21.2

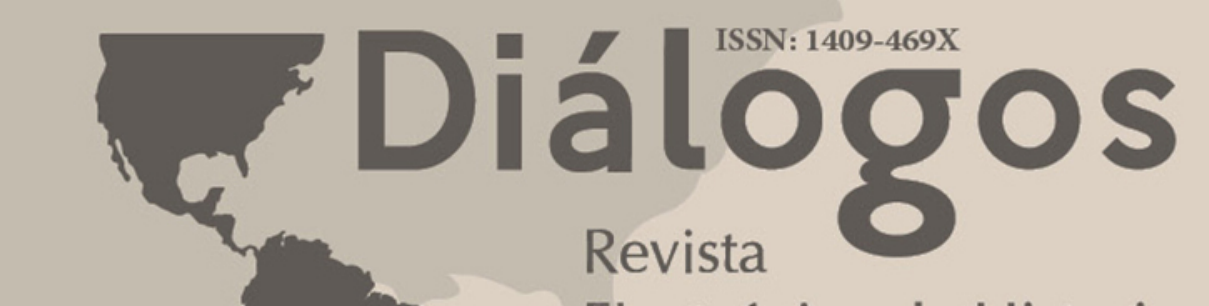

Electrónica de Historia

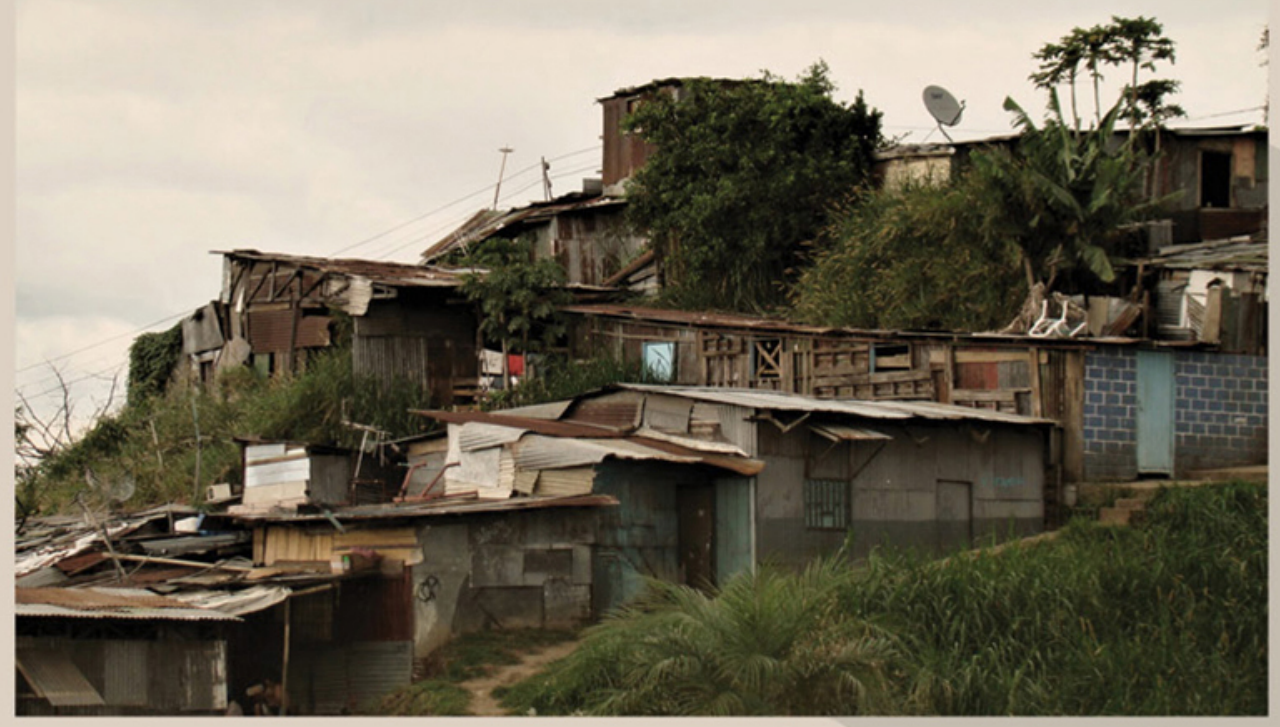

Centro de Investigaciones Históricas de América Central. Universidad de Costa Rica Julio-diciembre 2020

url: http://revistas.ucr.ac.cr/index.php/dialogos/index
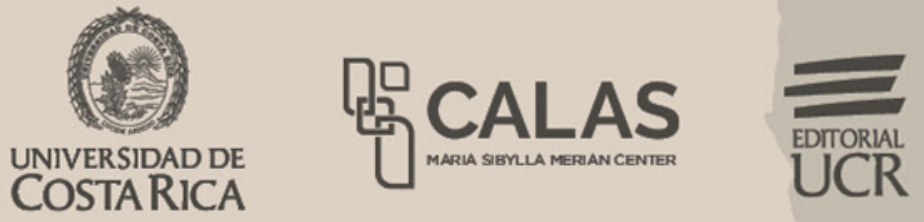


\title{
LA IMPUGNACIÓN A LA VIVIENDA-RANCHO EN LA CIUDAD DE CÓRDOBA (ARGENTINA) ENTRE LOS SIGLOS XIX Y XX
}

\author{
Ailen Suyai Pereyra \\ Cecilia Mercedes Quevedo
}

\begin{abstract}
Resumen
El trabajo analiza el contexto discursivo por el cual se moraliza la vivienda social mediante la desvalorización de la figura del "rancho" como habitabilidad dominante en barrios populares de la ciudad de Córdoba entre 1880 y 1920. En este marco, el artículo realiza una revisión del debate intelectual y político que instituye y delimita a la habitabilidad como esfera ideológica de intervención a sectores subalternos en Argentina. En esta labor analítica se propone tres vectores de análisis: los procesos de alterización enmarcados en la construcción del Estadonación de la región latinoamericana como antecedentes ideológicos al periodo; el rol del imaginario higienista en Argentina que ligado al positivismo operó como fundamento legítimo para intervenir el hábitat popular; y finalmente, como la condensación de los anteriores, las significaciones y valoraciones acerca de la vivienda-rancho en la provincia de Córdoba y sus implicancias en Pueblo Nuevo como un barrio popular. La estrategia metodológica se sustenta en el análisis a fuentes primarias, discursos políticos y documentos académicos elaborada por referentes de la elite médica del periodo. El resultado del artículo aborda las regulaciones estatales a la habitabilidad subalterna como ámbito sanitario, discurso político y problema público emergentes en las contradicciones entre acciones laicizantes y la estructuración colonial precedente.
\end{abstract}

Palabras clave: Estado-nación, higienismo, vivienda obrera, modernización, raza, clase social. 


\title{
THE OPPOSITION TO THE HOME-RANCH IN THE CITY OF CÓRDOBA (ARGENTINA) BETWEEN THE 19TH AND 20TH CENTURIES
}

\begin{abstract}
This work analyzes the discursive context in which social housing is moralized by devaluing the figure of the "ranch" as the dominant habitability in popular neighborhoods of the city of Córdoba between 1880 and 1920. In this framework, the article reviews the intellectual and political debate and delimits habitability as an ideological sphere of intervention for subordinate sectors in Argentina. In this analytical work, the article proposes three vectors of analysis: the processes of alterization framed in the construction of the nation-state of the Latin-American region as ideological antecedents to the period; the role of the hygienist imaginary in Argentina that, linked to positivism, operated as a legitimate foundation to intervene in the popular habitat. Finally, as the condensation of the elements above, the meanings and valuations about the ranch-house in the province of Córdoba and its implications in Pueblo Nuevo as a popular neighborhood. The methodological strategy is based on the analysis of primary sources, political discourses and academic documents that were prepared by referents in the medical elite of the period. The result of the article presents the state of regulations on subordinate habitability to sanitary scope, the political discourse, and emerging public problem in the contradictions between secularization actions and the preceding colonial structuring.
\end{abstract}

Keywords: Nation-state, hygienic, low-income housing, modernization, race, social class. 


\section{INTRODUCCIÓN}

Durante el periodo entre los siglos XIX y XX, la problemática de la vivienda popular se tornó un tópico central de discusión pública, instituyéndose en una nueva arena de intervención desde la órbita del Estado dentro de sociedades cada vez más complejas, populosas e industrializadas. En este marco, la población de Argentina se había incrementado como resultado de los procesos migratorios de ultramar pasando de dos millones en 1869 a ocho millones en 1914 (Boixadós, 2000a). El Estado asumió progresivamente la atención sobre la denominada "cuestión social" y el establecimiento de políticas concretas sobre las condiciones sanitarias ${ }^{1}$. Esto reorientó las acciones eclesiásticas que hasta entonces referían al control moral de las familias trabajadoras. El aumento poblacional, que se hacía más rápido que la oferta de vivienda e infraestructura de servicios, había generado que las enfermedades y epidemias se tornaran una de las principales preocupaciones institucionales en las ciudades.

En esta oportunidad, el trabajo analiza los discursos políticos de la provincia de Córdoba a partir de los pronunciamientos en el contexto de legislación sobre la vivienda obrera ${ }^{2}$, reconstruyendo aspectos hegemónicos ${ }^{3}$. Esta provincia es particularmente significativa dentro del denominado "interior" de Argentina por el peso simbólico que históricamente tuvo la Iglesia Católica desde el siglo XVI. No obstante, y para fines del siglo XIX, la provincia de Córdoba había comenzado un proceso identificado con el proyecto liberal que, confrontado con sectores católicos, ampliaba el horizonte de secularización de heterogéneos ámbitos problemáticos de la sociedad, entre ellos la vivienda de los sectores marginales. En el periodo analizado, Córdoba atravesó procesos de modernización (Ansaldi, 1997) que, condensando tradiciones intelectuales precedentes, buscaron transformar la imagen de ciudad colonial imitando diseños urbanísticos y sanitarios europeos con resultados contradictorios. En 1880, el asentamiento denominado "Pueblo Nuevo", que era incorporado al radio de la ciudad de Córdoba, fue un sitio testigo de la fuerza con que se aplicaron los discursos públicos sobre la regulación habitacional e higiénica sobre sectores populares. Sin embargo, esta tematización en torno a la vivienda popular no es reductible a un debate local, sino que se inscribe dentro de un repertorio heterogéneo y complejo de matrices ideológicas basadas en diferencias culturales y de clase social de ciertos pobladores urbanos, respecto a los cuales la elite política proponía diferenciarse.

En este sentido, el argumento del artículo se organiza de la siguiente manera. Por una parte, visitamos algunas discusiones latinoamericanas sobre interpretaciones racistas e inscritas en la moralización del trabajo y del trabajador (no indígena) que con el tiempo legitimaron la problematización en torno a la habitabilidad popular. Para ello establecemos algunas vinculaciones teóricas e historiográficas sobre la construcción del Estado-nación, producción de las identidades/alteridades y los discursos dominantes que ocupó, en esa diferenciación, la vivienda moderna. Por otra parte, abordamos la ideología positivista que, como nueva hegemonía basada en el higienismo social, definió el contenido simbólico de la nación, así como de 
los ámbitos provinciales como el que se trata de abordar. Por lo que se advierte que tanto el romanticismo, desde mitad del siglo XIX, como el positivismo, desde fines del XIX, elaboraron argumentos para la jerarquización racial de la población y de sus modos de vida basándose en características de los espacios domésticos. Finalmente, proponemos un vector de análisis local focalizado en las sentidos y valoraciones en la provincia de Córdoba sobre la política de salud pública y, dentro de éstas, el intento de erradicación del "rancho" comprendido como vivienda precaria. Ambas acciones estatales significaron a esta forma de habitación, generalizada en sectores trabajadores, desde su carácter inmoral, miserable o socialmente inapropiado. Esta particularidad permitió la delimitación del corpus analizado. Metodológicamente se utilizaron discursos políticos de autoridades cordobesas y documentos elaborados por los médicos y académicos que, como parte de una elite intelectual, estuvieron involucrados en el debate público del periodo, orientando las políticas públicas y los criterios de intervención en las habitabilidades populares.

El argumento general del artículo comprende que el discurso político, en el marco de pujas hegemónicas (Williams, 2000), constituye un mecanismo de traducción de las relaciones de dominación, subordinación y construcción de consensos sobre la representación política de las identidades y las alteridades. Como hipótesis, se sostiene que las intervenciones discursivas sobre el problema por la erradicación de la "mala vivienda" se tramó con signos de los discursos coloniales precedentes y nuevas valoraciones liberales e higienistas. En ellos podemos reconocer las condiciones contextuales por las cuales la élite urbana reproducía marcadores étnicos y de clase social, es decir, formas legítimas de inclusión y exclusión social en el escenario urbano que asocian el universo decimonónico cordobés con el racismo de clase (Balibar, 1991). Se basó en la perspectiva de Balibar (1991) para comprender cómo el racismo constituye un fenómeno social total que opera desde discursos y representaciones de profilaxis y segregación, permitiendo la construcción distintiva de identidades y alteridades como comunidades racializadas en contextos de explotación. Así, las formas físicas del rancho, como también la del conventillo ${ }^{4}$, progresivamente fueron simbolizadas como un peligro en sí mismo para el resto de la sociedad, consideración que se fue desplazando para juzgar a sus habitantes en términos raciales.

\section{MATRICES IDEOLÓGICAS: CIVILIZACIÓN Y BARBARIE COMO MITO FUNDACIONAL}

En las élites latinoamericanas, las representaciones de los intelectuales legitimando el orden social se difundieron en los discursos literarios, en las retóricas políticas, y posteriormente, en el conocimiento científico. El problema de las razas se instalaba como núcleo central de la erudición intelectual, haciendo que el racismo se convierta en la cualidad legitimadora pseudocientífica (Ansaldi \& Funes, 1994) de la matriz de los Estados latinoamericanos en su proceso de consolidación oligárquica. 
En la cultura política argentina, estos imaginarios giraron con base al mito fundante de civilización y barbarie y su innegable correlato geográfico entre Buenos Aires y el interior del país. El romanticismo "autóctono" proporcionó algunos de los referentes de la consolidación de la matriz de pensamiento a través de los cuales se han construido y demarcado las subjetividades nacionales y subalternas. Las obras literarias de intelectuales como Sarmiento y Alberdi sirvieron de fundamento de los discursos de la clase dominante y esa impronta impregnó la cultura política, ratificado como sentido común en la sociedad. Aunque desde aristas programáticas disímiles y adelantándose al positivismo, representaron un modo de percepción o un núcleo de pensamiento, un orden de ver y concebir, que se manifestó en los discursos dominantes y tecnologías específicas (principalmente a través de la escuela primaria por su capacidad de subjetivación).

A mitad del siglo XIX, la figura de Sarmiento permite caracterizar el espíritu de época por su estatus de referencialidad en la reproducción de categorías clasificatorias y morales sobre las diferencias sociales que luego tomó para sí el Estado nacional. En este sentido, la construcción eurocéntrica de lo autóctono se basa tanto en estéticas y moralidades contrapuestas como también en diacríticos y maneras de ser jerarquizadas. Por ejemplo, para Sarmiento, lo que daba sentimientos de "compasión y vergüenza" eran modos de vida y habitabilidades diferentes respecto a la cultura europea. En sus palabras:

Da compasión y vergüenza en la República Argentina comparar la colonia alemana o escocesa del sur de Buenos Aires y la villa que se forma en el interior: en la primera, las casitas son pintadas; el frente de la casa, siempre aseado, adornado de flores y arbustillos graciosos ... La villa nacional es el reverso indigno de esta medalla: niños sucios y cubiertos de harapos viven con una jauría de perros; hombres tendidos por el suelo en la más completa inacción; el desaseo y la pobreza por todas partes; una mesita y petacas por todo amueblado; ranchos miserables por habitación, y un aspecto general de barbarie y de incuria los hacen notables. (Sarmiento, 2007, p. 31)

La observación de lo doméstico desde entonces traslada las diferencias étnicas en torno a la habitabilidad como puntos sobre cómo operan los alcances de la civilización y la barbarie. La diferenciación entre "casitas" pintadas y aseadas con los "ranchos miserables" permanecerán indelebles en el imaginario popular y en la preocupación de los legisladores, como se verá más adelante.

Por su parte, Alberdi (1996) conduciría a una ideología proinmigratoria que proveería trabajadores para cumplir con uno de los factores de la producción que requirió el proyecto modernizador (Ansaldi \& Funes, 1994). Para este autor, la mejor constitución nacional que conviene al "desierto" argentino, es decir, aquella geografía habitada por sectoresétnicos indeseados, es la que sirve alos fines de hacerlo desaparecer como tal. Para el autor de Bases y Puntos de Partida para la Organización Política de la República Argentina (1996 [1852]), la extensión del territorio nacional obligaba a la imperiosa necesidad de poblar con inmigrantes europeos. 
La inmigración era clave en la intención de modificar el sustrato poblacional a partir de la mezcla de razas. En Alberdi (1996), la dicotomía central es el par "República posible/República real" donde la proyección de una "población deseada" implicaba que, posteriormente, serían seleccionados de los sujetos políticos. En ese binomio, el concepto de "trabajo" se define como actividad moral desde las características del determinismo racial. Alberdi (1996) entiende el par riqueza/pobreza como expresiones morales en función del trabajo y la ociosidad: define la riqueza moral a partir del trabajo y el ahorro, y la pobreza moral a partir de la ociosidad y la dilapidación. Así, la inmigración viabilizaría la cultura moderna, sus virtudes y laboriosidad, permitiendo alcanzar "la europeidad incompleta" (Margulis \& Belvedere, 1998,p. 116) de los argentinos.

Estas ideas cristalizaron en la élite del 1880, generación que protagonizó una epopeya desde los objetivos del liberalismo y el progreso. Desde entonces, Argentina se ubicaba como exportadora de materias primas a la vez que protagonizaba incipientes procesos de industrialización en centros urbanos. Desde entonces, y en el marco de la construcción del Estado-nación argentino, la reproducción del marco ideológico estuvo acompañada por profundas transformaciones sociales. Entre ellas se puede mencionar el ordenamiento legal y liberal del Estado, el crecimiento económico y demográfico, las políticas de educación pública, la inmigración a gran escala, los aportes del capital extranjero y, finalmente, las políticas urbanas e higienistas. El rápido éxito económico alcanzado demostraba las virtudes de la inserción del país en la división internacional del trabajo y en las modalidades de acumulación vigentes. A la vez, esto nutría a la cultura política de prejuicios étnicos y sociales respaldados en concepciones racialistas hacia amplios sectores poblacionales.

\section{LOS TRAZOS DEL POSITIVISMO Y EL PROBLEMA DE LAS RAZAS}

Si bien el liberalismo continuó siendo la ideología dominante, la década de 1880 significó una modificación en el panorama de las ideas hegemónicas: la transición final del romanticismo al positivismo. A principios del siglo XX, las prácticas políticas en dicho proceso se hallan indisociablemente unidas al conocimiento científico, brindando argumentos y representaciones para legitimar las propuestas de inclusión o exclusión a ciertos grupos sociales, aunque sin unanimidad (Villavicencio, 1999). Sólo con la ciencia moderna es posible que el racismo biológico, asociando rasgos físicos con cualidades morales, pueda convertirse en un discurso verosímil al clasificar y jerarquizar fenotipos "raciales". En este contexto, es posible concluir que América Latina funcionó como un laboratorio donde, en un mismo período de tiempo y hasta muchas décadas después, se llevaron a cabo diferentes $-\mathrm{y}$ homologables según los casos - prácticas basadas en el discurso racial, e incluso desde criterios estatalistas, raciológicos, deterministas y climáticos (González, 2000). 
En este periodo es posible analizar lo que para Nacach (2009) constituye un segundo momento de lo que puede englobarse como discurso racial, distinto al precedente por su base cientificista. La intelectualidad de Buenos Aires daba notable tratamiento al darwinismo en un contexto donde los extranjeros coetáneos atribuyeron excesiva devoción por el positivismo. Estos jóvenes intelectuales provenientes de clases medias fueron anexados por los representantes del Ejecutivo para articular una nueva élite al servicio del orden social ${ }^{5}$. Con ellos se fundó el carácter absoluto del sistema de ideas morales, proporcionando, desde el registro de la escritura, una dimensión fundante del nuevo saber sobre la sociedad.

El mismo sector, que a fines del siglo XIX buscó en el darwinismo social invocar la superioridad del hombre blanco durante el exterminio indígena, décadas más tarde se refugió científicamente en la eugenesia galtoniana para intervenir sobre el factor central de conflictos donde el peligro de la otredad, ya sea antes el indígena o luego el inmigrante, operó como un poderoso catalizador de intereses políticos (Miranda \& Girón Sierra, 2009). En los primeros años del siglo XX, el Ministro del Interior, Joaquín V. González, aplicaba en el senado elementos conceptuales relacionados a la eugenesia. Con estos argumentos daba cuenta de que Argentina era un país sin indios, asociando la noción de sociedad a una interpretación orgánico-biologicista.

Cuando comenzaba la crisis del paradigma oligárquico hegemónico, la heterogeneidad cultural de Argentina ofrecía a este tipo de intelectuales la representación de una nación fragmentada y en proceso de degradación. Ya era palpable el problema que la inmigración había acarreado en número y características. Por lo que las nociones vinculadas al modelo regeneracionista sirvieron de malla de fundamentos para explicar las consecuencias de la primera oleada inmigratoria europea que, contradiciendo las premisas de Sarmiento y Alberdi, finalmente había traído lo "no-deseado" del sur europeo. No sólo la diferencia aludía a los indígenas y a los negros, sino que se añadía el componente xenófobo al sujeto extranjero/anarquista $^{6}$ de origen europeo. Esta decepción en el potencial migratorio contribuyó a crear una crisis respecto al optimismo positivista. Esto significaba no tanto pérdida de fe en el progreso, aunque sí en el proceso civilizatorio como tal. De este modo, los males del mestizaje o "malos mestizos" necesitaron nuevas categorías (Nacach, 2009) haciendo que surja el par conceptual degeneracionismo/regeneracionismo ${ }^{7}$. Las perspectivas eugenésicas basadas en el par degeneración/regeneración (raza/ valores), la medición de los cuerpos tanto en la criminología, en la antropología y la frenología, protagonizan un nuevo giro legitimador ante una renovada invención de la nación frente al desorden de la problemática urbana.

El pensamiento eugenésico, junto con el concepto de la sociedad como un gran organismo, instaló en los ámbitos de salud pública europeos y luego americanos la preocupación que los grandes trastornos sociales de los siglos XIX y XX: la industrialización, el hacinamiento en grandes urbes, la miseria, nuevas formas de epidemias, grandes guerras, etc. (Álvarez Peláez, 1999). Se planteaba, como cuestión de Estado, los usos de teorías biológicas para exaltar los beneficios de la exclusión social. 
Las distancias objetivadas entre los grupos sociales se convertirían en un tópico urbanístico. Progresivamente, se fueron implementando políticas dirigidas a las principales entidades "amenazantes" para el futuro de la nación.

El problema de las razas comienza a tener un lenguaje médico, donde se trata a la sociedad como organismo enfermo y se objetiva al cuerpo discursivamente. La noción de "patología social" se instalaba en los discursos y las prácticas sociales, vinculando la genética con la identidad. Por ello, la corporalidad racializada debía ser el "objeto de estudio" incuestionable en la explicación científica. La clasificación y jerarquización racial era la estrategia positivista por excelencia, pues eran dos de las operaciones de naturalización producto de la "hermenéutica raciológica". El biologicismo, más que una simple aplicación de supuestos de la biología, era una metáfora vitalista de determinados valores sociales sexuados que transformaba los rasgos somáticos en síntomas de los caracteres psicológicos o culturales (Balibar, 1991).

Entre 1903 y 1913, en Bolivia, Perú, Argentina o Cuba, los intelectuales receptores del positivismo - cuyas obras en su mayoría fueron publicadas en países de Europa - explican el problema de las razas con relación al desorden político general o los "males latinoamericanos" (Ansaldi \& Funes, 1994). Por un lado, se ponía en evidencia los obstáculos al progreso en una nueva fase capitalista por el componente racial estructural y, por otro lado, las desviaciones respecto al propio orden provenientes del mismo proceso de modernización. A través de esta vinculación entre la genética de los grupos subordinados de cada región - las razas indígenas o negras, o ambas - y los frenos al desarrollo latinoamericano, se desligaba la responsabilidad a la oligarquía en proceso de debilitamiento. Los males latinoamericanos fueron entendidos, desde el lenguaje médico y sociológico, como un "destino" ineludible que eximía a la voluntad humana.

Por su parte, José Ingeniero fue uno de los mejores exponentes del positivismo de esta generación. Los trazos más fieles al positivismo recorren sus líneas en su obra El hombre Mediocre [1913] o famosa conferencia La formación de la raza argentina [1915]. En la primera obra, la dualidad entre la mediocridad y el ideal definirá la política como el ámbito de aquellos pocos hombres que, a diferencia de las multitudes mediocres, son capaces de desligarse de los determinismos porque su subjetividad es autónoma respecto a las elecciones y los actos. En la segunda conferencia, queda expuesto el argumento de la segregación a grandes sectores de la sociedad (incluso, un año antes se había opuesto al voto de las masas populares). Al plantear la formación de la nacionalidad argentina remite al concepto de "luchas de razas" antes que el de "lucha de clases". Pues la raza argentina constituyó una singular nacionalidad que no es herencia indígena ni mestiza, sino que ésta es custodiada por ciudadanos blancos y ajena a todo componente no-europeo (Margulis \& Belvedere, 1998). Para los pronósticos del autor, esa particular raza euro-argentina contribuyó a lograr el proceso de blanqueamiento de las ciudades a través de mecanismos de mestizaje y eso se percibe en las generaciones modernas que difieren de la estructura social colonial donde, por ejemplo, se sostiene que los negros ya se han extinguido. 
Los positivistas argentinos en su calidad de intelectuales desplegaron interés por la realidad concreta y los nuevos problemas sociales de las multitudes urbanas que ponen en riesgo la institucionalidad lograda. A la vez, estos van imaginando y proyectando un nuevo país desde la modernidad científica con base en discursos sobre las corporalidades y el color de la piel de las poblaciones, recreando el fundamento a las jerarquías sociales precedentes con nuevos argumentos y saberes legitimados. En esos planteos también habrá lugar para problematizar el hábitat popular desde discursividades médicas e higienistas de la provincia de Córdoba.

\section{EL HIGIENISMO COMO SABER LEGÍTIMO EN ARGENTINA}

Al revisar el espacio-tiempo que articula los siglos XIX y XX, se advierte que el rol del intelectual y de la reflexión latinoamericana de los problemas públicos encuentra en la ideología positivista un lenguaje para observar lo doméstico. En este horizonte, se recupera los aportes de Norbert Elías (1988) al tratar de considerar lo sucio y lo limpio dentro de la autocomprensión de occidente en los procesos de civilización ${ }^{8}$. Pues, el higienismo será el principal dispositivo de regulación (Foucault, 2006) en torno a la vivienda obrera como espacio de inscripción de lo limpio y lo sucio, que no siempre fue considerada en el análisis de las tecnologías de subjetivación dentro del imaginario civilizador.

Entre 1875 y 1885 , el higienismo adquirió prestigio mundial en un marco donde la ciencia médica descubrió la bacteria y las vacunas de Lister y Pasteur. En ese marco, el discurso de los profesionales de la higiene y la medicina recibe ineludible legitimidad "como práctica racional y científica que impone una intervención activa sobre la sociedad" (Cravino, 2016, p. 9). Oscar Terán (1987) afirmaba que la "cultura científica" es aquella difusa amalgama de intervenciones teóricas que reconocen el prestigio de la ciencia dando, de este modo, legitimidad a sus argumentos. Desde una concepción de la ciencia en sí misma, pero también en sus aplicaciones para "mejorar" el nivel de vida, impulsaron la implementación de disciplinas concretas que, quizás ninguna como el higienismo, coadyuvaron al control de lo social. Como expresaba David Viñas, "los señores ... se han hecho médicos en este momento y han tomado la palabra: la ideología higienista impregna toda la ciudad" (Viñas, 1995, p. 199).

Debido a los "males" del mestizaje, el Estado argentino asumió prácticas concretas: desde el control de epidemias, la separación de los hombres y mujeres a partir de un paradigma heterosexual, las medidas profilácticas y otras fórmulas de control, la provisión de agua y cloacas, hasta el control del nuevo electorado "radicalizado". Se definía la "Higiene Nacional" como el nuevo lenguaje que implicaba el control y la prevención de los males sociales y morales. Esta noción fue retomada posteriormente por las corrientes criminológicas en la primera década del siglo XX, donde la criminalidad se definió como enfermedad psicológica y moral que amenazaba la salud social. 
La mirada organicista proto-médica que se analiza en Sarmiento proporcionó muchas de las metáforas y formas de representación de los higienistas y criminólogos para explicar el proceso de modernización científica. Esta vez el modelo de análisis "salubre/insalubre" imaginó un nuevo enemigo invisible al cuerpo de la nación o el "cuerpo-país" al que se refirió Sarmiento (Salessi, 1995). El saneamiento de las ciudades, que en Sarmiento promovería a la circulación de los fluidos económicos, en los higienistas permitiría erigir un sistema de integración y articulación respecto a la idea del cuerpo-nación. En esa configuración, las transformaciones urbanísticas se centraron en "darle al centro un carácter de modelación de comportamientos" (Grosso, 2008, p. 24). Ante las crecientes migraciones del ámbito rural a las ciudades, la reconfiguración de los centros urbanos y las modificaciones de determinados espacios públicos acompañaron la nueva hegemonía cultural y su simbolización política. Por ejemplo, los mercados y los cementerios fueron reubicados en las periferias urbanas mientras que las plazas adquirieron jerarquía en los centros (Grosso, 2008).

Además del aluvión inmigratorio, la decepción, por su composición y consecuencias, y las complicaciones sociales que ocasiona el riesgo del sufragio universal profundizan la desconfianza por las "masas" que progresivamente llevaron al planteo de la cuestión social. En Argentina, José María Ramos Mejía es quien puso de manifiesto las problemáticas y el saldo de la política inmigratoria precedente que, a un ritmo acelerado, ha constituido una fuerza fenomenal sin raciocinio. En Las multitudes argentinas [1899], y analizando las multitudes desde la época virreinal hasta su presente, Ramos Mejía utilizó herramientas de Le Bon para desarrollar una psicología social a partir del carácter biológico de las multitudes.

En 1890, más del $70 \%$ de la deuda externa argentina había sido para financiar las obras de salubridad, demostrando la hegemonía de la disciplina en el proyecto modernizador. En 1892, Ramos Mejía como presidente del Departamento Nacional de Higiene extiende el higienismo como política "nacional" al interior del país. En este sentido, con el proceso de modernización, se organizó en Argentina una sociedad disciplinaria esta vez a través de discursos y dispositivos de la higiene montados en una red de vigilancia. La modalidad principal con la que funcionó este sistema disciplinario estuvo organizada en función de la observación y la recolección de datos a través de sedes sanitarias del Departamento Nacional de Higiene en diferentes puntos del país. Se mantenían bajo la mirada desde los barcos hasta ciudades, barrios, escuelas, fábricas, talleres, ranchos y conventillos: fue la puesta en práctica del panoptismo argentino (Salessi, 1995).

Los nuevos saberes, con sus metáforas patológicas e higienistas, trataban de disciplinar un espacio social cuya masificación comenzaba a visibilizar fisuras. Siguiendo el trabajo de Jorge Salessi (1995) desde finales de las últimas décadas del siglo XIX, la disciplina interviene en la realidad social formulando dispositivos para extender el poder central desde los espacios del Estado y a partir de las tecnologías totalizantes e individualizantes. Allí, la clínica, como espacio de observación científica, aportó una mirada médica sobre lo social y su tratamiento. 
Durante este lapso de tiempo, la construcción de la identidad fue a través de la sublimación de las relaciones sociales mediante el vínculo de ciudad como ámbito con la cultura urbana con el saber. Se reafirmaba el papel de la ciencia al relacionarla con la forma física de la ciudad como fiel expresión de sus ideas y como lugar por excelencia del progreso donde la arquitectura posee su propio espacio discursivo. El ámbito urbano no sólo fue el hervidero de la "cuestión social", sino que, como bien demostró Vallejos (2007) analizando la fundación de la ciudad de La Plata en 1882, floreció producto de los usos de la ciencia que demarcaba formas de vivir en la ciudad. A través de la diagramación urbana, el imperativo positivista finisecular y la cultura urbana se volvían un valor en sí mismos. Así, las estrategias del liberalismo argentino no remiten únicamente a las modalidades represivas, sino que su éxito se debió a la sedimentación de la cultura urbana socialmente legitimada.

\section{LA HEGEMONÍA HIGIENISTA EN CÓRDOBA: ENTRE LA MORAL Y LA MODERNIZACIÓN}

En Argentina, los "conventillos" de la ciudad de Buenos Aires se asumieron como la situación generalizada en el país. Estas habitaciones populares eran piezas o cuartos alquilados a inmigrantes de origen europeo generalmente, para una familia o un grupo de hombres solos. En esas interpretaciones, la "huelga de las escobas" de 1907 (Suriano, 1984), debido a la suba de los alquileres en los conventillos, fue interpretada como una de las expresiones de la organización política de las familias de inmigrantes en el periodo. Tomando distancia respecto a estos abordajes, el objetivo del trabajo analiza los argumentos del periodo por fuera de explicaciones concebidas para la ciudad de Buenos Aires y más allá de la situación de los "conventillos" en la ciudad puerto. Como también ha pasado con otras temáticas relativas a la organización del sistema sanitario, Buenos Aires "erróneamente ha sido tomado como el paradigma del desarrollo histórico argentino" (Carbonetti, 2005,p. 90). En el interior del país existen muchas distancias con aquella mirada centralista e incluso menor grado de industrialización, quizás los "ranchos" hayan sido el escenario doméstico más representativo de los nuevos procesos sociales y económicos en esos territorios.

Como región mediterránea, y con el cambio de siglo, la ciudad de Córdoba (Figura 1) comenzaría lentamente una preliminar etapa industrial de la mano de la vorágine de los proyectos de modernización provincial (Ansaldi, 1997). Dejando atrás el lugar eminente de centro religioso propio de la estructuración colonial, los procesos de industrialización se remontan a la incorporación del ferrocarril y los circuitos económicos ligados al modelo agroexportador que éste habilita en torno a 1870 y 1880 . En lo político, Córdoba estuvo estrechamente asociado a la hegemonía del nuevo discurso liberal ${ }^{9}$, el cual consideraba al Estado como prioritario tanto para el progreso económico como el cuidado de la sanidad pública. 
Las instituciones de salud en la provincia de Córdoba comenzaron a transformarse cuando tomó el poder una élite liberal en lo económico y conservadora en lo político. En este contexto, el gobernador Miguel Juárez Celman, quien llevó a cabo el proceso modernizador, consideraba al abrir las sesiones legislativas de 1881 que:

La higiene pública, ... es una de las más importantes obligaciones, que en las sociedades civilizadas, pesan sobre la administración general y municipal, que tienen a su cargo, todas las funciones inherentes al régimen policial. Ella... sin embargo no había sido organizada hasta el presente en la Provincia. (Juárez Celman citado en Carbonetti, 2005,p. 96)

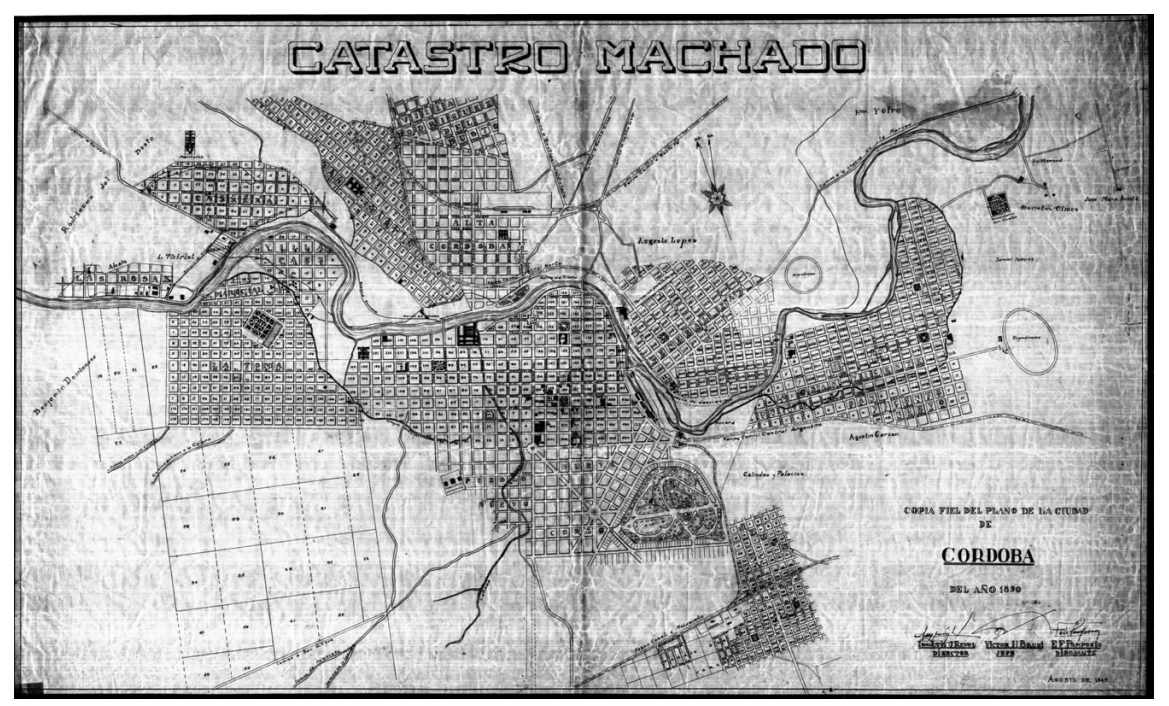

Figura 1. Machado, A (1890). Plano de la Ciudad de Córdoba. Fuente: Archivo Histórico Municipal. Mapa

Precedente a las políticas de "Higiene nacional" de Ramos Mejía, las medidas en el espacio público cordobés daban cuenta de una nueva hegemonía propuesta en clave de "civilización". Para Adrián Carbonetti, la conformación del sistema de salud pública de la provincia de Córdoba se remonta a 1880, "año en que se crea el Consejo de Higiene y comienza un proceso de medicalización del sector salud" (2005, p. 95). Su hipótesis radica que el sistema de salud se desarrolló con escasa intervención del nivel provincial en cuanto a la organización de instituciones y estructuras asistenciales. Por el contrario, las asociaciones de beneficencia desde sectores provenientes de la iglesia, los Estados nacional y municipal, así como la Universidad Nacional de Córdoba mediante la Facultad de Medicina fueron activos promotores de políticas al respecto. Esto consagró una legislación oficial contradictoria: buscaba reglamentar la práctica de la salud pública, pero autorizaba su ejercicio a instituciones no estatales.

Por otro lado, el mundo doméstico iba concediendo relativa significatividad a las prácticas de la higiene familiar. La historiadora Cecilia Moreyra postula que "en siglo XIX no sólo se incrementó notablemente la cantidad de artefactos destinados 
a lavar el cuerpo, sino que esos objetos tendieron a ser cada vez más específicos" (2017, p. 213). Identificando el espacio doméstico cordobés, la autora aborda la presencia o ausencia de ciertos objetos materiales y sitios destinados a la higiene del cuerpo, lo que permite dimensionar cómo se suscitan procesos de cambios en los hábitos de aseo. Moreyra (2017) concluye que la apropiación de objetos y prácticas de higiene no pueden ser generalizables a todas las clases sociales, pero sí permiten evidenciar los alcances que tuvo la importancia del aseo personal y colectivo en el periodo. De hecho, de su estudio sobre las prácticas cotidianas es posible tener en cuenta los efectos del desplazamiento de las sensibilidades a propósito de la naturalización de estas maneras de hacer en los espacios domésticos, sensibilidades que también organizaban las políticas hacia los sectores subalternos.

Más allá de las nuevas prácticas sociales, las políticas desde el Estado municipal de la ciudad de Córdoba fueron el punto de partida para dimensionar los impulsos pioneros del higienismo como modo de intervención racional. Desde la planificación urbanística hacia fines del siglo XVIII, las ordenanzas municipales se habían encargado de regular distintas prácticas en el espacio público entendiéndose como cuestiones de salubridad pública: erradicar curtiembres cercanas a los cursos de agua, eliminación de entierros en el centro, la estructuración de un sistema de riego de cultivos, construcciones de caminos, la sistematización del cauce del Río Suquía, el amojonamiento de terrenos, el desplazamiento del uso rural de la tierra, entre otros. Además, hacia 1880 se iniciaron las primeras obras de cañerías domiciliarias para mejorar el abastecimiento de agua expandiendo el acceso doméstico (Boixadós, 2000b). De esta manera, se buscaba establecer y diferenciar una traza urbana respecto a una periferia abocada a actividades rurales. A la vez, la construcción e identificación de la salud pública, en este sentido, tuvo que ver con la erradicación del trabajo del ámbito doméstico.

No obstante, las intenciones de ordenar y limpiar el espacio urbano en Córdoba datan de 1839 y 1840 según diversas fuentes historiográficas. Entre 1860 y 1880, el sector comprendido de Pueblo Nuevo junto con las otras tres regiones (La Bomba, el Infiernillo y El Abrojal) tenían un doble carácter basado en la existencia de un área considerada "moderna" y otra "atrasada". El sector poseía algunos servicios urbanos básicos que dotaban del sentido moderno para la época: una plaza para las carretas, tranvía, más tarde alumbrado, comercios, entre otros. No obstante, eran las autoridades las que consideraban que aún prevalecía el carácter "popular y atrasado". Esta distinción dio el pie para que el Intendente Luis Revol (1887-1890) incorporara la zona dentro del radio municipal con el objetivo de sanear los alrededores del centro. En este marco, la configuración espacial Pueblo Nuevo como barriada popular fue producto de la interacción, disputa y solapamiento de relaciones sociales en el contexto de ciudad, enmarcadas en el proceso de modernización de la ciudad capital de la provincia.

Los argumentos por los cuales se definía a la higiene como la necesidad de prevención de los males sociales y morales en Córdoba operaron en dos sentidos: por un lado, como el lugar de asepsia social que perduraría en el tiempo justificando a las políticas habitacionales implementadas para "obreros ${ }^{10} " ; y$, desde argumentos higienistas, 
la delimitación de un enemigo público desvalorizando formas de construcción habitacional que no se correspondía a la utilización de materiales industriales; es decir, la construcción del "rancho" como antagonista de la salud pública cordobesa, tal como se verá más adelante. En concreto, y siguiendo las tendencias higienistas de la época, el intendente Revol en el año 1888 decide crear el primer plan de viviendas que buscaba erradicar el rancho y dar mejores condiciones de vida a la población trabajadora. Las acciones del intendente de la ciudad de Córdoba en materia habitacional estaban orientadas a "brindar casas para obreros, intervención enmarcada en el discurso del higienismo social liberal y por lo tal, combatida por los sectores conservadores de Córdoba" (Boixadós, 2000a, p. 5) principalmente a través de la prensa.

En ocasiones, la prohibición de construir ranchos desde las normativas municipales confrontaba abiertamente con las posiciones religiosas, que apelaban a la inviolabilidad de la propiedad privada. Como expresa Boixadós (2000a), mediante la órbita de la vivienda se controlaba la vida privada del beneficiario, la permanencia del hogar y el valor de la familia. Además, esta estrategia ideológica mantenía a los trabajadores más arraigados en el trabajo y el ahorro, aquellos valores que proponía Alberdi a mitad del siglo XIX como se veía en el primer apartado, que en opciones políticas cercanas al anarquismo o al socialismo. En Córdoba, y en épocas donde la Encíclica Rerum Novarum del Papa León XIII acercaba a círculos católicos con demandas obreras, este tipo de opciones políticas nunca prosperaron a diferencia de lo ocurrido en Buenos Aires (Suriano, 1984).

El plan de Casas de Inquilinato de Revol se implementó en parte de espacio público y en la Plaza de las Carretas. El proyecto se insertó en Pueblo Nuevo buscando limpiar la imagen moral y física de la población trabajadora cumpliendo con ciertos requisitos. El Estado planificó unas 84 viviendas unifamiliares de una superficie cubierta de $66 \mathrm{~m}^{2}$ con ciertas características físicas: un jardín compartido, patio individual, dos habitaciones, comedor, letrina, agua corriente y sumideros. Indudablemente, el plan se llevaría a cabo en dos manzanas de Pueblo Nuevo concebidas para que contrasten con el atraso en materia urbanística de la barriada. La política pionera en el país fue posible gracias a la bonanza económica que vivió la provincia y el país en la década.

Para el Intendente Revol, la población trabajadora era construida como un solo colectivo, identificado como una familia pobre y vulnerable frente al capital. Ante esta situación y recepcionando las preocupaciones higienistas, al Estado municipal

no le queda otro medio que el de fomentar la construcción de casas de inquilinato, por medio de primas, garantías del capital, exoneración de impuestos, etc., o el de construirlas por su cuenta, haciendo que el monto del alquiler sea lo más módico posible, apenas en relación con el servicio del capital invertido ... servirán de albergue conveniente a numerosas familias, con atenuación de las cargas que sobre ellas pesan, abonando un alquiler modesto, en armonía con sus recursos (Revol citado en Boixadós, 2000a, p. 6). 
Estas edificaciones proponían una interesante estrategia de control moral de la población popular en relación con pestes, disturbios y promiscuidad. Estas viviendas otorgaban condiciones de ventilación, disminución del hacinamiento y comodidad. Ciertamente, el plan de viviendas como estrategia sanitaria combinaba dos posiciones hegemónicas y contradictorias del periodo: por una parte, otorgaba una morada higiénica con espacios privados para sus miembros y en condiciones salubres que no pusieran en riesgo al resto de la sociedad. Por otro lado, estaba orientado a contener a las familias que se adhirieran a ciertos principios morales y demostraran capacidad de ahorro mediante un trabajo disciplinado. La vivienda estatal, de esta manera, simbolizaba la higiene buscada, pero a la vez condicionaba el estatus de "familias obreras de moralidad insospechada" (Boixadós, 2000a, p. 8) para quienes fueran sus ocupantes.

Sin embargo, el proyecto de Revol no se concretó en su totalidad, pero las instalaciones sirvieron para alojar algunas familias durante las inundaciones producidas por el arroyo La Cañada en los años 1890 y 1894. No obstante, en el periodo existían varios factores que aparecían fundamentando la prevención y la sanidad poblacional. Por una parte, el gradual crecimiento poblacional de Córdoba y la escasa planificación de la extensión urbana había dado lugar a una disposición desordenada de la población. Los diagnósticos referían a las condiciones de habitabilidad física y moralmente negativas para los habitantes. No sólo representaban desventajas higiénicas, sino que carecían de reglas de construcción. Por otro lado, "Córdoba tenía altas tasas de mortalidad por las gastroenteritis, tuberculosis y difteria que encontraban asilo en las aguas servidas y los excrementos que se desparramaban por cualquier parte" (Valdemarca, 2016, p. 117). Además, en 1906 hubo un brote de cólera en Tucumán y se preveía que pudiera extenderse a Córdoba que, entre 1867 y 1868 , había perdido alrededor del $10 \%$ de su población por esa epidemia.

\section{LA VIVIENDA-RANCHO COMO HABITABILIDAD INMORAL}

En Córdoba, la cuestión social se constituyó como un tópico dual: por una parte, la salubridad pública se aplica a toda la ciudad y al ámbito de las viviendas de los obreros; y por la otra, la formación de organizaciones de defensa de los intereses y derechos de los trabajadores tanto desde ámbitos religiosos como desde acciones estatales. Así, la intervención sobre la población trabajadora se volvía indispensable para generar condiciones de funcionamiento del sistema político. La problematización y los debates legislativos de la llamada vivienda obrera, se cree, responden a diversos sentidos y valores que, en este periodo histórico, no pueden ser solo reductible al déficit habitacional en el periodo (Page, s/f).

A principios de siglo XX, las propuestas que abordaban el saneamiento de la vivienda de los trabajadores también provenían de la clase alta asociada a los círculos médicos, por lo que no sólo estas iniciativas estuvieron amparadas por los preceptos 
morales de las organizaciones religiosas. Entre 1900 y 1920, Córdoba fue escenario de la convergencia entre el sector católico (desde la Asociación de Artesanos de San José y las Conferencias Vicentinas) y los destacados referentes del higienismo e impulsores de una legislación sobre la vivienda obrera. Los claros exponentes de las legislaciones fueron los médicos Félix Garzón Maceda y Juan Cafferata, cuyas trayectorias forman parte de la profesionalización de la medicina y, por ende, de la concentración de poder desde la conformación de una élite médica ${ }^{11}$. Asimismo, como parte de su generación de intelectuales e higienistas, se encargaron de asentar sus posiciones desde el registro de la escritura y recurrente participación en círculos médicos, congresos o eventos científicos.

El primero, Garzón Maceda, fue diputado de la provincia en tres oportunidades y legisló la Ley de Casas para Obreros que fue presentada en el Congreso Nacional en 1906. Con el tiempo se convirtió en el historiador de la medicina en la provincia. Garzón Maceda fue un estudioso de la práctica de "proto-medicato", que consistía en regular el ejercicio de la medicina de acuerdo con las leyes de la monarquía, y un defensor de su ineficacia ante la emergencia de las instituciones modernas estructuradas en el auge del higienismo y la medicalización social. Hasta 1878 que se funda la Facultad de Medicina de la Universidad Nacional de Córdoba, en ese momento el cuidado de la salud o el asunto de la vida y de la muerte eran incumbencias de curanderos, brujos o chamanes de las poblaciones indígenas (Rodríguez, 2007). El conocimiento positivo, es decir sistemático, objetivo y fundante, debía guiar las políticas sanitarias dando cuenta del carácter pre-científico de la totalidad de los saberes hasta entonces. En este marco de institucionalización del higienismo, en 1917, Garzón Maceda se pronunciaba de este modo de las "rancherías barrosas y pajizas":

\footnotetext{
De donde resulta que allí donde, como en Córdoba, se advierte la acumulación de casas materialmente anti-higiénicas aun fuera de la zona de las rancherías barrosas y pajizas, los porcentajes de morbilidad y mortalidad son mayores; $\mathrm{y}$ todas las enfermedades epidemiales o contagiosas tienen arraigo y se extienden a la manera de las gramíneas rastreras. (Garzón Maceda, 1917,p. 496)
}

El segundo, Juan Cafferata fue un diputado nacional de la provincia de Córdoba e impulsor de la Ley 9677 de 1915 que crea la Comisión Nacional de Casas Baratas (Ballent \& Liernur, 2014). También se desempeñó como profesor sustituto y delegado de la Facultad de Medicina de la Universidad Nacional de Córdoba. El médico higienista tenía autoridad suficiente para exponer las consecuencias negativas de la existencia de ranchos y conventillos. Desde este estatus, Cafferata expuso en distintos auditorios universitarios sobre los alcances sociales de la "mala vivienda". En 1916, bajo el subtítulo "el rancho", este médico mencionaba a propósito del "enjambre de construcciones miserables" que observaba un viajero al ingresar a la ciudad de Córdoba: 
Cuando el viajero se aproxima a Córdoba por sus grandes vías ferrocarrileras, experimenta primero una impresión ingrata. Las pintorescas barrancas de suburbios, donde los accidentes del terreno brindan a1 propietario motivos de aprovechamiento excepcionales (testigo el Jardín Zoológico), están incultas y desnudas. Algunos arbustos y plantas rastreras, a guisa de jardines, adornan pobremente la aridez de las laderas arenosas y un enjambre de construcciones miserables, verdaderas chozas de salvajes, se levantan en las pequeñas mesetas, en las lomas o en las cañadas, sin orden y sin plan, a la voluntad de sus dueños y a merced de su capricho. La estética, la higiene, la profilaxis, se encuentran, si existen, en estado absolutamente rudimentario. El mismo espectáculo se presenta en los distintos rumbos. (Cafferata, 1916, p. 350)

En el contexto de la modernización urbanística de Córdoba, las "chozas de salvajes" que describe Cafferata son similares a los "ranchos miserables" a las que se refería Sarmiento. Desde licencias literarias y estetizaciones, ambos intelectuales remiten a que la forma de habitar producía un sentimiento de vergüenza y desagrado. En 1917, el mismo médico refiere a la "mala vivienda" como un verdadero foco infeccioso en otra conferencia:

La mala vivienda es con el alcoholismo la gran productora de tuberculosis. La
vivienda estrecha, oscura, superpoblada, donde no llegan ni el rayo de sol, ni
el aire oxigenado; donde viven hacinadas las familias en una atmósfera física
y moralmente irrespirable, verdaderas estufas de cultivo que hacen germinar,
prosperar y multiplicarse los agentes de la destrucción del individuo y de la
especie. (Cafferata, 1917, p. 362)

Las iniciativas legislativas como la Ley de Casas para obreros (o Ley Garzón Maceda), la Ley de Casas Baratas y la Protección de la iniciativa privada para la construcción de la casa propia y económica se proyectaron desde Córdoba al resto del país. La principal premisa de esta generación de médicos higienistas y legisladores era que "la vivienda es el primer problema social" (Cafferata, 1916, p. 344). En el plano público, la solución al problema de la habitabilidad obrera podía ser conducida a través de una planificación estatal de las viviendas para obreros, más allá de la órbita municipal que veíamos con el proyecto pionero de Revol. La legislación nacional de viviendas, en primer lugar, se reducía a la eliminación de casas precarias, pues la ecuación entre alcoholismo, tuberculosis y hacinamiento fueron identificadas como causantes de la elevada cifra de mortalidad y, por ende, un verdadero peligro para el resto de la sociedad decente. En este sentido, una habitación higiénica era considerada suficiente para la reproducción saludable de un trabajador y de su familia, pero propiciando originariamente la impugnación a los ranchos como forma dominante de la habitabilidad subalterna cordobesa.

Dentro del horizonte moral y pregnancia de discursos católicos, Cafferata hacía referencia a la familia nuclear como elemento moral y deseable porque "la vida de familia, en el verdadero concepto del hogar, desaparece sin la vivienda adecuada" (Cafferata, 1916, p. 344). No obstante, como se decía antes, lo más significativo de la producción discursiva en esta generación de médicos era que la desvalorización en torno a la figura del rancho condensa expresiones ideológicas propias del legado de Sarmiento, tal como veíamos en el primer apartado, o incluso 
de significantes como los ligados al "orden evolutivo". Este médico higienista expuso en una conferencia en 1917:

El tipo de la vivienda primitiva y en el orden evolutivo de la habitación podía figurar con ventaja como el primer eslabón después de la choza del aborigen. $\mathrm{Su}$ aspecto es característico y su construcción elemental. Un poco de madera rudimentaria labrada, paja y barro, son sus materiales. Por excepción algunos llevan techo de fierro galvanizado. Su índole primitiva le permite emplazarse en cualquier parte, en hacinamiento, sin consultar reglas de construcción ni de higiene. (Cafferata, 1917, p. 366).

En este sentido, se asocia la condición material de la vivienda a la pertenencia cultural propia de una alteridad radical. Así, el problema de lo que denominaban "mala vivienda" se articulaba a un discurso moralizador desde el ejercicio del racismo de clase (Balibar, 1991), lo que permite introducir componentes de etnización dentro de las relaciones asimétricas. De esta manera, en las conferencias de los médicos higienistas, el sujeto trabajador fue descrito frecuentemente desde diacríticos étnicos desde los cuales se organizan las representaciones de profilaxis y segregación más amplias. El rancho era símbolo del espacio de una población indeseada y sucia - por la presencia del barro y las prácticas supuestamente promiscuas - describiéndolo como un tipo de habitación "primitiva" posterior a "la choza del aborigen". Pues, la tipología y las características del rancho significaron el nivel de atraso que actualizaba el núcleo de pensamiento del romanticismo en el siglo anterior y posibilitaba clasificaciones sociales basadas en la posible regeneración a través de los valores.

Además, la división que había fundado la sociedad industrial desde fines del siglo XIX basada en la separación entre la fábrica y el hogar no podía permitir al obrero "emplazarse en cualquier parte". En efecto, las nuevas condiciones requeridas para el desarrollo de relaciones capitalistas de producción no sólo veían en el rancho un problema sanitario, sino un obstáculo al afincamiento para la asalarización por el carácter "primitivo" de prácticas nómades, asociadas implícitamente a las indígenas "salvajes". Así, la vivienda popular, más allá de instituirse como lugar ascético para la inmunidad del resto de la sociedad, operaba como el lugar de encierro de cuerpos indeseados para la mirada de una élite médica encargada de regular lo social.

\section{CONCLUSIONES}

Desde fines del siglo XIX, el solapamiento de imaginarios y discursos políticos respecto a los ámbitos domésticos y la cuestión habitacional reproducen desigualdades a la par de procesos de diferenciación cultural, normalización y disciplinamiento que no siempre fueron vislumbrados por la historia social en el "interior" del país. Entre 1880 y 1920, el higienismo social no sólo proporcionó una interpretación verosímil de la realidad social en distintas regiones de Latinoamérica, sino que es la 
nueva hegemonía (Williams, 2000) que fundamenta la matriz de dominación política y de jerarquización social en Argentina.

De este modo, en el artículo se aborda el contexto y las construcciones ideológicas que permearon los discursos médicos y políticos que legitimaron la necesidad de transformar la habitabilidad de los sectores subalternos. Se parte desde tres claves de lectura: como construcción cultural e histórica en los inicios de la industrialización en Latinoamérica, desde los imaginarios asociados al romanticismo y al positivismo dentro de los procesos de dominación y de construcción higienista del Estado-nación en Argentina; y como la amalgama de discursos legítimos que tuvieron lugar durante la modernización y el inicio del problema de la vivienda en Córdoba como provincia mediterránea.

En este recorrido, los discursos políticos y médicos sobre la erradicación del "rancho" condensan dos procesos sociales del periodo: por una parte, la primigenia industrialización y racialización del proletariado urbano; por otra parte, la emergencia de una élite médica como nueva estructura de dominación en la ciudad de Córdoba. Dentro de los procesos de modernización provincial, la producción de consensos políticos se advierte en los valores y roles estatales en torno a la vivienda popular. En efecto, el saber médico e higienista converge con el dominio católico-colonial en el debate público cordobés: la impugnación del rancho reúne tanto argumentos de salud pública como de moralidad social. Estos criterios morales que buscaban acercar posiciones con el orden católico no se detuvieron con el tiempo dentro de las estrategias estatales de resolución de la cuestión habitacional. Tampoco lo hizo la construcción de ranchos que, como tipología de vivienda precaria, demostraban las desigualdades de la clase trabajadora cordobesa y los niveles socioeconómicos que no mejoraron en el tiempo a pesar de la beneficencia católica o asistencia estatal.

En este marco, referir a las iniciativas estatales sobre la construcción de viviendas obreras en Pueblo Nuevo posibilitó explorar la producción de los dos mundos culturales en constante tensión ideológica en ciudad de Córdoba. Uno de ellos atrasado, hediento, con una población popular de hábitos inmorales y pertenencias ligadas al pasado indígena; en oposición, se encontraba un sector moderno, pulcro, compuesto por familias acomodadas y membresía a círculos de la alta sociedad, con títulos universitarios y pertenencias de origen europeos. Mientras el primer grupo popular es objeto de las intervenciones higienistas y moralizaciones como colectivo de trabajadores disciplinados y beneficiados con una vivienda, mucho antes que en el escenario de Buenos Aires; el segundo tenía competencias culturales y socioeconómicas que les permitían hacer juicios de valor y tomar decisiones acerca de la planificación urbana que requería la modernización provincial. Entre ambos mundos culturales, el espacio doméstico y la consideración de las normas de habitabilidad aparecen como patologías y discursos racializadores que legitiman intervenciones estatales, con pretensiones liberales, pero con valores católicos sobre ciertos grupos sociales. 


\section{NOTAS}

1 El concepto de la "cuestión social" remite a las consecuencias sociales negativas, laborales e ideológicas producto del proceso de industrialización y urbanización desde fines del siglo XIX.

2 Según el historiador Waldo Ansaldi, "la instalación de fábricas en Córdoba significa la aparición de la industria capitalista, mas no un proceso de industrialización en sentido estricto" (Ansaldi, 1997, p. 52). Debido a esta particular dinámica industrial, es necesario aclarar que la referencia a la categoría "obrero" o "vivienda obrera" remite al uso pragmático que están explícitamente presentes en los discursos que se analizaron. En este sentido, se referirá a vivienda popular, obrera o para trabajadores como sinónimo, aludiendo a aquellas habitabilidades destinada para sectores subalternos en general y diferenciando de los procesos de estructuración social suscitado en estos escenarios recién a mitad de siglo XX.

3 La localización de la provincia de Córdoba se ubica en la región centro de la Argentina y a 700 kilómetros de Buenos Aires. Con un pasado de conquista española y colonial ligado al Virreinato del Perú mediante la Gobernación de Tucumán, desde el proceso independentista forma parte del "interior" en contraposición a la ciudad de Buenos Aires. Los sectores conservadores de la provincia estuvieron representados por élite del poder eclesiástico y la tendencia restrictiva del sistema político que se vería atacado por el liberalismo de fines del siglo XIX y luego por el radicalismo del siglo XX. En el proceso político cordobés tuvo sus singularidades: tanto los partidos conservadores como el Partido Radical fueron filtrados por sectores católicos.

En Córdoba, la noción de "conventillo" difiere de la concepción porteña popularizada en Buenos Aires. En esta región remite a la construcción de varios ranchos en un terreno para alquiler (Valdemarca, 2016).

En Buenos Aires, algunos referentes fueron José Ingeniero, Leopoldo Lugones, Ricardo Rojas, Enrique del Valle Iberlucea, entre otros. Algunas figuras, como Ingeniero, encontraron en el positivismo un instrumental para defender un socialismo haciendo conciliar de manera original a Marx (referente del socialismo) y a Comte (referente del positivismo). A nivel internacional las referencias son más amplias. La noción de Gustav Le Bon de "alma nacional", es decir, la realidad social etnométrica, demostraba que las características psicológicas eran heredadas. Si pensamos en Carlos Octavio Bunge, este autor sintetiza la herencia que ocasionó las experiencias de caciquismo y caudillismo en la composición racial Argentina: la pereza criolla, arrogancia mulata y tristeza samba (Ansaldi \& Funes, 1994).

La Ley 4.144 de Residencia se sanciona en 1902, autoría de Miguel Cané. Habilitó al gobierno a expulsar a inmigrantes sin juicio previo ante la inminencia de las ideas anarquistas, dando cuenta de la emergencia del "delincuente", a través del nuevo proceso de significación que vinculó el inmigrante con el anarquista y una nueva otredad del proceso de ciudadanía como de inclusión/exclusión.

7 Se trataba de una nueva matriz científica legitimadora concerniente a la sociología basada, en su mayoría, en los referentes raciales europeos, como Taine, Le Bon y Galton y teorías criminológicas, como la de Lombroso.

El filósofo Rodolfo Kusch (2007) recurre a la metáfora referida al hedor y a la pulcritud para referirse la cultura popular argentina. Las reflexiones del autor nos sirven para pensar las articulaciones entre el Estado y los comportamientos controlados. Las metáforas permiten aludir a la capacidad de normalización de las políticas de Estado-nación que operaron respecto a la identificación de una barbarie hedienta como alteridad siempre singular, no sólo los 
«indios asquerosos» que identificó Sarmiento (2007). La dicotomía categorial y sensitiva de Kusch (2007) concibe una historia nacional a "contrapelo" ahondando allí donde la hegemonía del imaginario civilizador y el orden racionalizado encontró en lo urbano su posible la continuidad.

9 La Provincia de Córdoba ocupó un lugar central en el proceso de consolidación del régimen que se instauró a partir de 1880 con la afirmación del Partido Autonomista Nacional como partido hegemónico en la Argentina: Julio Argentino Roca (1880-1886) y, el ex gobernador de Córdoba, Miguel Juárez Celman (1886-1890). Luego de la revolución de 1890, se desata una crisis a partir de la renuncia de Juárez Celman a la presidencia de la Nación donde la Provincia fue perdiendo el poder que ostentaba. En este marco, el General Roca incrementó su poder hasta finalizar su segundo mandato en 1904.

10 Denominado así por el mismo Revol. Las casas de inquilinato serían destinadas a población de bajos recursos o población obrera.

11 Siguiendo a Rodríguez (2007), la particularidad de la élite cordobesa pasaba por la concentración de cátedras en la Facultad de Medicina de Córdoba, la participación del Consejo Provincial de Higiene, el desempeño de cargos políticos en la provincia y la municipalidad de Córdoba; y las membresías en instituciones como el Círculo Médico de Córdoba creado en 1910.

\section{REFERENCIAS}

Alberdi, J. B. (1996). Bases: y puntos de partida para la organización política de la República Argentina. Venezuela: Biblioteca Ayacucho.

Álvarez Peláez, R. (1999). Dossier: Estudios sobre eugenesia. Revista Asclepio, (2), 5-9. DOI: 10.3989/asclepio

Ansaldi, W. (1997). Una modernización provinciana: Córdoba, 1880-1914. Revista Estudios, (7 y 8), 51-80. DOI: $10.31050 / 1852.1568 . n 7-8.13950$

Ansaldi, W. y Funes, P. (1994). Patologías y rechazos. El racismo como factor constitutivo de la legitimidad política del orden oligárquico y de la cultura política latinoamericana. Nueva Época, 1(2), 193-229.

Ballent A. y Liernur, J. (2014). La casa y la multitud. Vivienda, política y cultura en la Argentina moderna. Argentina: Fondo de Cultura Económica.

Balibar, E. (1991). El racismo de clase. En Balibar, A. y Wallerstein, I. (Eds.), Raza, nación y clase (pp. 313-333). Argentina: IEPALA.

Boixadós, M. C. (2000a, agosto). La vivienda como parte de las políticas de salud del municipio de Córdoba a fines del siglo XIX y principios del XX. Ponencia presentada en Primeras Jornadas de Historia Regional Comparada, Brasil. Recuperado de http://cdn.fee.tche.br/jornadas/1/s9a5.pdf

Boixadós, M. C. (2000b). Las Tramas de una ciudad, Córdoba entre 1870 y 1895. Élite urbanizadora, Infraestructura, poblamiento. Argentina: Ferreyra editor. 
Cafferata, J. (1916, julio). La vivienda obrera en Córdoba. Apuntes presentados en la Congreso Americano de Ciencias Sociales, San Miguel de Tucumán.

Cafferata, J. (1917). El saneamiento de la vivienda en la profilaxis contra la tuberculosis. Relación entre las condiciones de la vivienda y la mortalidad por tuberculosis en el Municipio de Córdoba. Apuntes presentados en la Conferencia Nacional de Profilaxis contra la Tuberculosis.

Carbonetti, A. (2005). La conformación del sistema sanitario de la Argentina. El caso de la Provincia de Córdoba, 1880-1926. Dynamis: Acta Hispanica ad Medicinae Scientiarumque. Historiam Illustrandam, 25, 87-116. Recuperado de https://ddd.uab.cat/record/28018?ln=ca

Cravino, A. (2016). Historia de la vivienda social. Primera parte: del conventillo a las casas baratas. Vivienda y Ciudad, 3, 7-24. Recuperado de https://revistas.unc.edu.ar/index.php/ReViyCi/ article/view/16262

Elías, N. (1988). El proceso de la civilización. Investigaciones sociogenéticas y psicogenéticas. México: Fondo de Cultura Económica.

Foucault, M. (2006). Vigilar y castigar. El nacimiento de la prisión. Argentina: Siglo Veintiuno Editores.

Garzón Maceda, F. (1917). La medicina en Córdoba. Apuntes para su historia (Tomo III). Argentina: Talleres gráficos Rodríguez Giles.

González, H. (Comp.). (2000). Historia crítica de la sociología argentina. Los raros, los clásicos, los científicos, los discrepantes. Argentina: Colihue.

Grosso, J. L. (2008). Semiopraxis en contextos interculturales poscoloniales. Cuerpos, fuerzas y sentidos en pugna. Revista Espacio Abierto Cuaderno venezolano de Sociología, (2), 231-245. Recuperado de https://dialnet.unirioja.es/ejemplar/206283

Kusch, R. (2007). América profunda. Argentina: la Fundación Ross.

Margulis, M. y Belvedere, C. (1998). La "racialización" de las relaciones de clase en Buenos Aires: genealogías de la discriminación. En Margulis, M. y Urresti, M. (Eds.), La segregación negada: cultura y discriminación social (pp. 79-122). Buenos Aires: Biblos.

Miranda, M. y Girón Sierra, A. (2009). Cuerpo, biopolítica y control social. América Latina y Europa en los siglos XIX y XX. Argentina: Siglo XXI.

Moreyra, C. (2017). Cultura material e higiene cotidiana en la Córdoba del Ochocientos. Anuario de Estudios Americanos, 74(1), 211-234. DOI: 10.3989/aeamer.2017.1.08

Nacach, G. (2009). Cuestión de paradigmas: conquista, representaciones y pensamiento racial en la Pampa y Patagonia argentina (1860-1915). Ea-jornal, 1(2). Corpus ID: 188859571. Recuperado de www.ea-journal.com

Page, C. (s/f). Las primeras viviendas obreras. Recuperado de http://www.carlospage.com.ar/wpcontent/2008/06/Page-Vivienda-obrera-en-cordoba.pdf 
Rodríguez, M. L. (2007). La élite médica y su definición interna como grupo de poder en Córdoba, 1878 y 1923. XI Jornadas Interescuelas/Departamentos de Historia. Departamento de Historia. Facultad de Filosofía y Letras. Universidad de Tucumán, San Miguel de Tucumán. Recuperado de https://www.aacademica.org/000-108/890

Salessi, J. (1995). Médicos maleantes y maricas: Higiene, criminología y homosexualidad (Buenos Aires: 1871-1914). Argentina: Edita Beatriz Viterbo.

Sarmiento, D. (2007). Facundo. Argentina: Terramar.

Suriano, J. (1984). La huelga de inquilinos de 1907 en Buenos Aires. En Armus, D. (Comp.), Sectores populares y vida urbana. (pp. 56-67) Argentina: Clacso.

Terán, O. (Comp.). (1987). Positivismo y nación en la Argentina. Argentina: Puntosur.

Valdemarca, L. (2016). Feos, sucios y peligrosos o sus cambiantes combinaciones según los tiempos. Travesía, Suplemento. VII Reunión del Comité Académico de Historia, Regiones y Fronteras, (pp. 113-127). Recuperado de http://www.travesia-unt.org.ar/suplemento.php?nlibro=21

Vallejos, G. (2007). Escenarios de la cultura científica argentina. Ciudad y universidad (1882-1955). España: CSIC, Colección Estudios sobre Ciencia

Villavicencio, S. (1999). José Ingeniero y el imaginario positivista de la ciudadanía. En Jacques, R.; Quiroga, H.; Villavicencio, S. y Vermeren, P. (Comp.), Filosofías de la Ciudadanía. Sujeto Político y democracia. Argentina: Homo Sapiens.

Viñas, D. (1995). Literatura argentina y política I: De los jacobinos porteños a la bohemia anarquista. Argentina: Sudamericana.

Williams, R. (2000). Marxismo y Literatura. España: Península. 